\title{
Necrotising pneumonia is an increasingly detected complication of pneumonia in
} children

\author{
G.S. Sawicki ${ }^{*,+}$, F.L. Lu*, ${ }^{*,}$ C. Valim ${ }^{\#}$, R.H. Cleveland ${ }^{\star}$ and A.A. Colin*
}

ABSTRACT: Necrotising pneumonia (NP) is a severe complication of community-acquired pneumonia characterised by liquefaction and cavitation of lung tissue. The present study describes the epidemiology, aetiology, management and outcomes of children hospitalised with NP over a 15-yr period.

A retrospective observational study of NP cases was conducted from January 1990 to February 2005 analysing clinical presentation, laboratory data, hospital course and long-term follow-up.

A total of 80 NP cases were identified, with the number of detected cases increasing from 12, in the period 1993-1996, to 40 in the period 2001-2004. In total, 69 (86\%) cases had pleural effusion with a low pH (mean 7.08) and 38 (48\%) patients had positive cultures, with Streptococcus pneumoniae as the predominant organism. Recently, other organisms, most notably methicillinresistant Staphylococcus aureus, emerged. Patients had prolonged hospitalisations (median 12 days). A total of 69 patients required pleural interventions and those receiving chest drainage alone had similar outcomes to those managed surgically. All patients had full clinical resolution within 2 months of presentation.

Necrotising pneumonia has increasingly been identified as a complication of paediatric pneumonia. Streptococcus pneumoniae remains the predominant organism, but since 2002, different bacteria have been isolated and the age range of cases has broadened. Despite the serious morbidity, massive parenchymal damage and prolonged hospitalisations, long-term outcome following necrotising pneumonia is excellent.

KEYWORDS: Cavitatory pneumonia, empyema, necrotising pneumonia, pleural effusion, pneumonia

omplications of community-acquired pneumonia in children include pleural effusion, empyema, lung abscess, pneumatocele and necrotising pneumonia (NP). NP, also termed cavitary pneumonia or cavitatory necrosis, has been associated with poor clinical outcomes in adults and when initially described, this complication was thought to be extremely rare in children. The first case series of NP including four children was published in 1994 [1] and subsequently there have been several reported small case series of paediatric NP [2-6] including a recent report on epidemiological observations [7].

Even though the diagnosis of NP can be suspected by plain chest radiography, the frequent presence of a dense lobar consolidation and pleural effusion may obscure proper definition and chest computed tomography (CT) scanning is needed for a more definitive diagnosis. Radiographic criteria for NP include the loss of normal pulmonary parenchymal architecture and the presence of areas of decreased parenchymal enhancement, representing liquefaction, that are progressively replaced by multiple small air or fluid filled cavities $[1,8]$. The pathophysiology of NP is thought to be one of massive pulmonary gangrene, tissue liquefaction and necrosis [9] but the precise pathways leading to this massive damage have not been well established. The radiological diagnosis of NP has been correlated with such pathological findings [10]. Prior reports have focused on NP caused by Streptococcus pneumoniae [11], although other bacterial organisms, including Staphylococcus aureus and Mycoplasma pneumoniae, have reportedly led to NP $[12,13]$. The increasing number of case reports of NP has coincided with several studies of childhood pneumonia, which report trends of increasing incidence of complicated pneumonia with parapneumonic effusions $[14,15]$. While mention is made of NP in these

\section{AFFILIATIONS}

*Division of Respiratory Diseases, Dept of Medicine,

\#Clinical Research Program and Division of Gastroenterology and Nutrition, Dept of Medicine,

'Dept of Radiology, Children's Hospital Boston, Harvard Medical School, Boston, MA, USA.

${ }^{+}$Both authors contributed equally to this study.

CORRESPONDENCE

G. Sawicki

Children's Hospital Boston 300 Longwood Avenue Boston MA 02115 USA Fax: 16177300097 E-mail: gregory.sawicki@ childrens.harvard.edu

Received:

August 032007

Accepted after revision: January 102008

\section{SUPPORT STATEMENT}

Some of the results of this study have been previously reported in the form of an abstract at the $7^{\text {th }}$ International Conference on Paediatric Pulmonology (CIPP VII; Montreal, Canada) July 2006.

STATEMENT OF INTEREST None declared. 
studies, the related role of NP to this changing trend has not been analysed.

The purpose of the present, retrospective, observational study was to review the cases of NP among children hospitalised at the Children's Hospital, Boston (Harvard Medical School, Boston, MA, USA) over a 15-yr period and describe: 1) the epidemiology, causative organisms and key clinical and laboratory characteristics of children hospitalised with NP; 2) the management strategies and presence of complications in children with NP; and 3) report on the long-term clinical outcomes for children with NP.

\section{METHODS}

\section{Case selection}

Cases of NP among patients hospitalised at Children's Hospital Boston from January, 1990 to February, 2005 were identified retrospectively using an electronic database of the Dept of Radiology. All cases were identified by searching for the term "necrotising" in reports of thoracic CT scans of hospitalised patients and reports with terms such as "no evidence of necrotising pneumonia" were excluded. CTs of all cases were reviewed and included for analysis when the CT revealed segments of lung showing multiple areas of lung parenchyma containing air, air and fluid, or nonenhancing fluid surrounded by contrast enhancing lung parenchyma without a defined rim of enhancement [8]. Cases of solitary cavitation surrounded by a well-defined enhancing rim of lung were excluded, since this could represent a lung abscess.

\section{Data collection}

A retrospective hospital chart review was conducted using a standardised data collection form. Patients with nosocomial pneumonia and those with pre-existing lung or cardiac disease were excluded from subsequent analysis. For each patient, demographics, laboratory results, microbiological culture data and clinical information originally obtained at the time of admission, during the admission and at any subsequent outpatient follow-up visits at the institution, were recorded. For the present study, a febrile day was defined as any 24-h period during which the patient had a recorded temperature $\geqslant 38^{\circ} \mathrm{C}$. Hypoxia was defined as any recorded oxygen saturation of $<90 \%$ by pulse oximetry, measured on room air. The study was approved by the Children's Hospital Boston Institutional Review Board.

\section{Statistical methods}

Demographic, clinical and laboratory variables were summarised by standard descriptive statistics. Comparisons between groups based on clinical presentation, interventions and complications were performed using paired t-tests where means are reported, Wilcoxon tests where medians are reported and Chi-squared tests where proportions are reported. Two-sided p-values $<0.05$ were considered to be statistically significant.

\section{RESULTS}

A total of 80 cases of community-acquired NP were identified during the 15-yr study period. There were no cases identified in the period 1990-1993 and the number of detected cases increased over time from 3 cases $\cdot \mathrm{yr}^{-1}$ in 1993-1996 to 14 case$\mathrm{s} \cdot \mathrm{yr}^{-1}$ in 2003-2004 (fig. 1).

\section{Clinical presentation and radiographical findings}

The median age at presentation was 3.6 yrs (interquartile range (IQR) $0.25-19$ yrs; table 1). The majority of patients had no significant prior medical history; only $14(18 \%)$ patients had a reported history of asthma or wheezing and nine (11\%) had a reported history of recurrent otitis media. With the exception of two cases, these children had no known underlying immunological diseases. One patient had a known underlying immune deficiency (Schwachman-Diamond Syndrome) and another patient was diagnosed with chronic granulomatous disease subsequent to presentation with NP. Figure 2 shows representative CT images from one patient, a 4-yr-old previously healthy female, and demonstrates the radiographic criteria used in identifying NP, as well as the time-course of the disease.

Almost all patients had fever (96\%) and cough (84\%), with a mean onset of symptoms 9 days prior to the hospitalisation. Other constitutional symptoms such as vomiting, abdominal pain and chest pain were only reported by a minority of patients. A total of $54(74 \%)$ patients received at least one dose of oral antibiotics prior to their hospitalisation. For these patients, the mean duration (range) of pre-admission antibiotics was 3 (0-24) days.

\section{Laboratory and microbiological characteristics}

The predominant laboratory features (table 2) were leukocytosis (mean white blood cell count $18.4 \times 10^{3}$ cells $\cdot \mu \mathrm{L}^{-1}$ ), bandaemia (mean 9\%), anaemia (mean serum haemoglobin $10.4 \mathrm{mg} \cdot \mathrm{dL}^{-1}$ ) and hypoalbuminaemia (mean serum albumin $2.0 \mathrm{mg} \cdot \mathrm{dL}^{-1}$ ). An appreciable pleural effusion on radiograph or CT scan was seen in $69(86 \%)$ patients. Pleural fluid analysis revealed a low $\mathrm{pH}$ (mean 7.08), a low glucose (median $10.0 \mathrm{mg} \cdot \mathrm{dL}^{-1}$ ) and a high cell count (median 9,600) with neutrophil predominance. Positive microbiological identification was obtained in 38 (48\%) cases (fig. 1). A total of 19 cases had positive pleural fluid cultures and

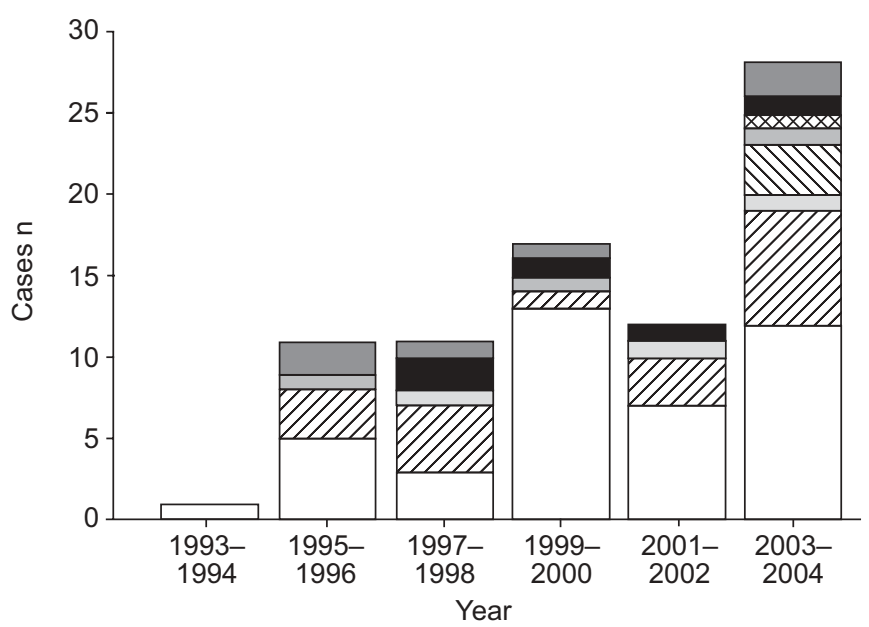

FIGURE 1. Necrotising pneumonia cases in the period 1993-2004. Within each 2-yr interval, the distribution of culture-negative cases and culture-positive cases, with each organism separately identified, is shown. $\square$ : culture negative; $\mathbb{Z}$ : Streptococcus pneumoniae; : : methicillin-sensitive Staphylococcus aureus; $\mathbb{\mathbb { N }}$ : methicillin-resistant Staphylococcus aureus; $\mathbf{1}:$ Fusobacterium spp.; $\mathbf{0}$ : Pseudomonas spp.; ש: Staphylococcus spp. non-aureus; $\mathbf{0}$ : other Streptococcus spp. 


\begin{tabular}{|c|c|c|}
\hline \multirow[t]{2}{*}{ TABLE 1} & \multicolumn{2}{|c|}{$\begin{array}{l}\text { Demographic and clinical characteristics of the } \\
80 \text { children with necrotising pneumonia }\end{array}$} \\
\hline & & Summary statistics \\
\hline \multicolumn{3}{|c|}{ Demographic characteristics } \\
\hline Age yrs & & $3.6(2.4-6.2)$ \\
\hline Sex male & & $42(53)$ \\
\hline \multicolumn{3}{|l|}{ Race } \\
\hline White & & $38(48)$ \\
\hline Black & & $2(3)$ \\
\hline Hispanic & & $11(14)$ \\
\hline Asian & & $7(9)$ \\
\hline Other & & $4(5)$ \\
\hline Undocun & inted & $18(23)$ \\
\hline \multicolumn{3}{|c|}{ Medical history } \\
\hline Asthma or & heeze & $14(18)$ \\
\hline Bronchopu & onary dysplasia & $1(1)$ \\
\hline Cerebral pa & & $1(1)$ \\
\hline$>3$ episod & of otitis media & $9(11)$ \\
\hline \multicolumn{3}{|c|}{ Clinical presentation } \\
\hline Fever & & $76(96)$ \\
\hline Height of $f$ & er ${ }^{\circ} \mathrm{C}$ & $38.8 \pm 0.9$ \\
\hline Cough & & $66(84)$ \\
\hline Chest Pain & & $14(18)$ \\
\hline Vomiting & & $27(34)$ \\
\hline Shortness & breath & $16(20)$ \\
\hline Abdominal & & $25(32)$ \\
\hline Days with $\varsigma$ & nptoms pre-hospitalisation & $8.8 \pm 5.3$ \\
\hline \multicolumn{3}{|c|}{ History of medical care } \\
\hline Received a & ibiotics pre-admission & $54(68)$ \\
\hline Days of an & iotics pre-admission & $3.0 \pm 4.3$ \\
\hline Hospitalisa & In (at another hospital) & $31(39)$ \\
\hline
\end{tabular}

Data are presented as $n(\%)$, median (interquartile range) or mean $\pm \mathrm{SD}$.

five had positive pleural fluid latex agglutination studies for Pneumococcal antigen. Other aetiological diagnoses were based on positive blood or sputum cultures. Receiving any antibiotics pre-admission was not associated with a reduction of positive microbiological identification (48 versus 56\%; $\mathrm{p}=0.5)$. S. pneumoniae was identified in $18(22 \%)$ cases (13 by positive culture and five via positive pleural latex agglutination studies). The diagnoses based on latex agglutination all had negative pleural fluid cultures. The absolute number of Pneumococcus spp. cases per year was similar throughout the study period. Review of the antibiotic susceptibility patterns of the positive Pneumococcal cultures did not reveal any penicillin-resistant Pneumococcus spp.

Since 2000, the incidence of other causative organisms, including methicillin-sensitive $S$. aureus, methicillin-resistant S. aureus (MRSA), Fusobacterium, Pseudomonas aeruginosa and Streptococcal sp. such as S. milleri, has increased. All cases of MRSA were detected after 2003.

\section{Outcomes during hospitalisation}

The median length (IQR 9-17) of hospitalisation was 12 days, ranging from 3-84 days (table 3). During the hospitalisation, the median duration of fever was 6 days (IQR 3-9) ranging from 1-28 days. There were no statistically significant differences in the median number of febrile days between patients based on antibiotic pre-treatment ( 5 versus 7 days; $\mathrm{p}=0.18$ ) or those presenting with pleural effusion (6 versus 4.5 days; $p=0.24)$. There were no deaths in the cohort. Different types of antibiotic regimens were used as initial empiric treatment: including penicillins, cephalosporins, vancomycin and clindamycin, and in cases with positive cultures, antibiotic regimens were tailored to specific organism susceptibilities. All patients were treated with prolonged courses of antibiotics (median (range) 27 (3-95) days).

\section{Management of patients presenting with pleural effusion}

Of the 69 patients presenting with pleural effusions, 47 (68\%) had placement of a chest tube or pigtail catheter for pleural drainage, $16(23 \%)$ underwent a combined surgical intervention with chest tube placement and only six (9\%) patients underwent thoracentesis alone (table 3). In those receiving surgical intervention, $12(17 \%)$ underwent video-assisted thoracoscopic surgery, three $(4 \%)$ had an open thoracotomy and pleural decortication and one $(1 \%)$ patient had a wedge resection of the affected parenchymal lobe. The median duration of pleural drainage was 6 days (IQR 5-10, range 152 days) and did not differ based on the type of intervention (table 4). The number of febrile days also did not differ based on the type of intervention $(p=0.65)$; however, the median length of stay was significantly longer in patients requiring chest drainage only or surgery (14 and 14.5 days, respectively) compared with patients having only a thoracentesis (11 days) or those without pleural effusion ( 6 days; $\mathrm{p}=0.0002$ ).

Of the patients with pleural drainage, 10 (15\%) developed a bronchopleural fistula (BPF) defined by persistent air leaks noted in the medical record lasting $>24 \mathrm{~h}$. Patients who developed BPF were older (median age 6.0 versus $3.5 \mathrm{yrs}$; $\mathrm{p}=0.06$ ) than patients without BPF. The presence of a BPF was associated with a longer time of pleural drainage (median 14 versus 6 days; $p=0.0007$ ) and a longer length of stay (median 19 versus 13 days; $p=0.01$ ). All of the $B P F$ cases were managed conservatively without operative repair.

\section{Long-term outcomes}

A total of eight $(10 \%)$ patients required readmission (six due to persistent fever) within 2 weeks following their initial hospital discharge. Three patients developed a small pneumothorax, none required intervention. Of the total cohort, 64 patients were seen post-discharge in the paediatric pulmonary clinic at the Children's Hospital (median follow-up time 6 months), all with complete clinical resolution of symptoms reported within 2 months of discharge. Of these, 12 patients had pulmonary function testing (PFT) performed; eight $(67 \%)$ had normal PFTs, three patients had a mild obstructive defect and one had a mild restrictive defect. Follow-up imaging studies included chest radiographs and, in a few cases, chest CT scans (fig. 3), all of which showed marked improvement within 6 months with near normalisation of pulmonary parenchymal structures.

\section{DISCUSSION}

In the present study, 80 paediatric cases of NP presenting over 15 yrs at a single institution have been identified. With the exception of two cases, these children had no significant 

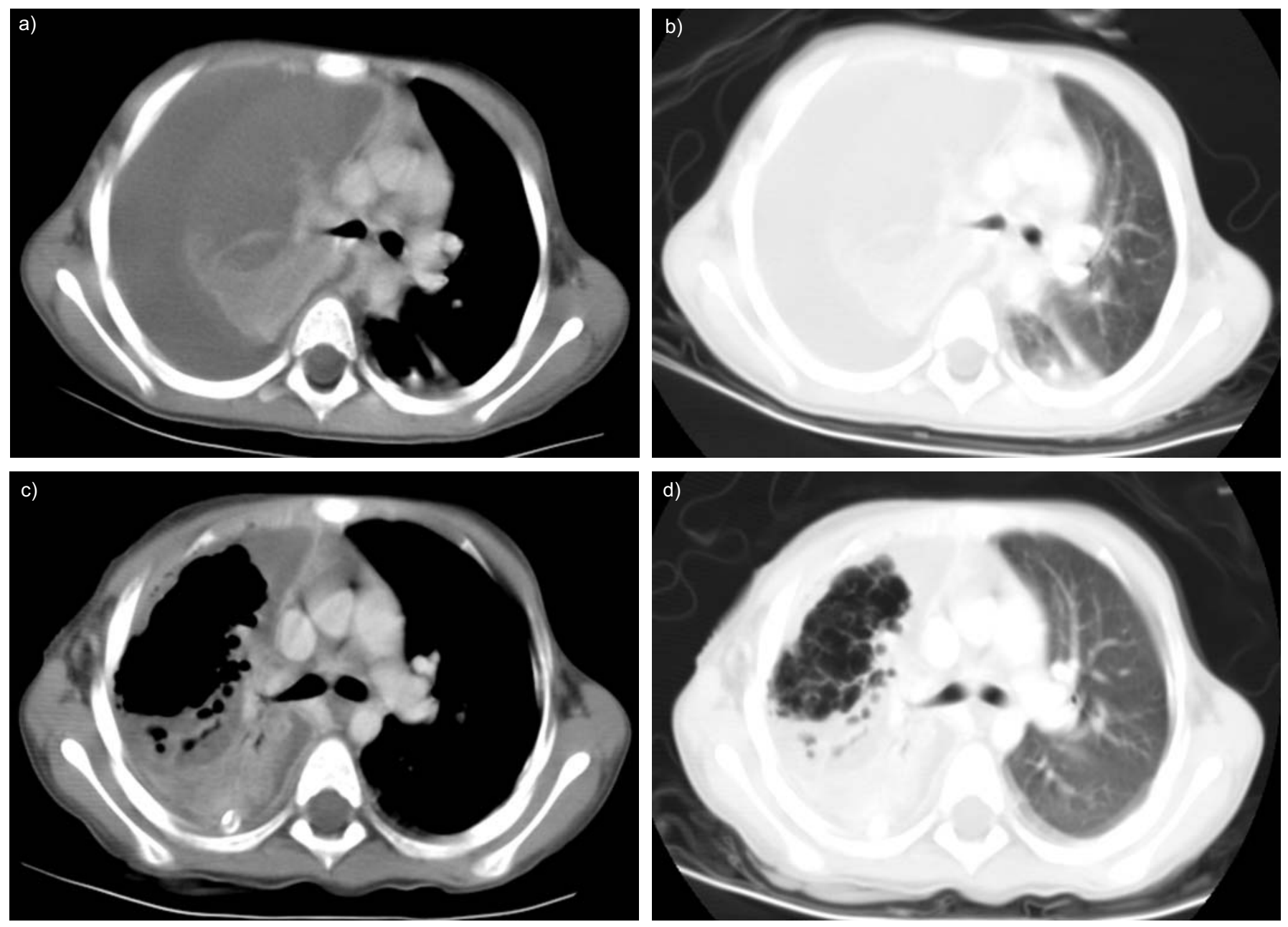

FIGURE 2. Representative chest computed tomography (CT) images from a 4-yr-old female hospitalised with necrotising pneumonia (NP). The mediastinum (a, c) and lung (b, d) CT windows are shown at the same anatomic level. a, b) Early CT findings of NP including the liquefaction of lung parenchyma and development of a pleural effusion. c, d) A CT scan was performed 3 days later showing the development of multiple air filled cavities within the lung parenchyma.

underlying comorbid conditions, such as immunological disease or a history of prior infections. Their course was associated with significant radiographical evidence of lung damage and prolonged hospitalisations. The detection of NP in children increased over time. S. pneumoniae was the most commonly identified organism but other causative bacteria were cultured. Association with empyema was common. Despite the significant short-term morbidity, both clinical and radiographical resolution has been observed over time.

The presence of NP as a unique entity within the spectrum of complicated pneumonia has been previously analysed in a few small series of patients [1-4], with the largest series at one institution including 17 patients [2]. Using a retrospective case definition for NP based on CT diagnoses, 80 cases were identified. Even accounting for the possibility of underreported cases due to reliance on a CT definition for diagnosis, the present series represents the largest group of paediatric NP cases systematically described to date.

The age range and demographic features of the present patients are similar to other published studies of children with complicated pneumonia [14-16]. Most children had developed symptoms of fever and cough for $\leqslant 1$ week prior to their hospitalisation, indicating that the parenchymal damage in NP occurs rapidly. Transition from liquefaction to cavitation within $48 \mathrm{~h}$ has been observed via CT scan. Although the retrospective nature of the present analysis results in missing laboratory data for some of the cases, the abnormal laboratory findings of an elevated white blood cell count and low haemoglobin that were observed in many of the patients, have been previously reported in children with complicated pneumonia and NP [2, 16]. A markedly decreased serum albumin level was found in many patients, potentially secondary to loss of protein into the affected lung parenchyma and pleural fluid or possibly due to protein losing enteropathy [17]. The pleural fluid characteristics associated with NP in the present study, particularly the low $\mathrm{pH}$, have also been associated in other studies with increased complications [18-20]. The majority of the present patients had a persistent fever and, given the frequently sterile cultures and predominantly sensitive organisms, the present authors assume that the reason for the fever is not related to the lack of effectiveness of eradication of bacteria but rather to the presence of pyrogenic products of inflammation and tissue destruction [20]. 


\begin{tabular}{|c|c|c|}
\hline \multirow[t]{2}{*}{ TABLE 2} & \multirow[b]{2}{*}{ Summary statistics } & \multirow[b]{2}{*}{ Subjects } \\
\hline & & \\
\hline \multicolumn{3}{|l|}{ Serum laboratory values } \\
\hline WBC count $\times 10^{3}$ cells $\mu \mathrm{L}^{-1}$ & $18.4 \pm 8.9$ & 77 \\
\hline Haemoglobin $\mathrm{g} \cdot \mathrm{dL}^{-1}$ & $10.4 \pm 1.6$ & 74 \\
\hline Haematocrit \% & $30.9 \pm 4.4$ & 75 \\
\hline Albumin $\mathrm{g} \cdot \mathrm{dL}^{-1}$ & $2.0 \pm 1.6$ & 40 \\
\hline $\begin{array}{l}\text { Erythrocyte sedimentation } \\
\text { rate } \mathrm{mm} \cdot \mathrm{h}^{-1}\end{array}$ & $96.7 \pm 22.9$ & 21 \\
\hline C-reactive protein $\mathrm{mg} \cdot \mathrm{dL}^{-1}$ & $13.3 \pm 9.3$ & 20 \\
\hline \multicolumn{3}{|l|}{ Pleural fluid values } \\
\hline $\mathrm{pH}$ & $7.08 \pm 0.33$ & 50 \\
\hline Glucose $\mathrm{mg} \cdot \mathrm{dL}^{-1}$ & $10.0(2.0-65.5)$ & 44 \\
\hline $\mathrm{LDH} I U \cdot \mathrm{L}^{-1}$ & 2810 (1413-9530) & 43 \\
\hline Cell count $\times 10^{3}$ cells $\cdot \mu \mathrm{L}^{-1}$ & $9.6(1.2-56.2)$ & 54 \\
\hline Pleural neutrophils \% & $70 \pm 23.4$ & 52 \\
\hline \multicolumn{3}{|l|}{ Culture results } \\
\hline $\begin{array}{l}\text { Any bacterial organism iden- } \\
\text { tified }\end{array}$ & $38(48)$ & 80 \\
\hline
\end{tabular}

Data are presented as mean $\pm S D, n$, median (interquartile range) or $n(\%)$. WBC: white blood cell; LDH: lactate dehydrogenase.
Over the 15 yrs covered by the present study, an increase in the absolute numbers of NP diagnosed in the Children's Hospital was observed. The rise is likely due to a combination of increased recognition of NP as a specific entity and heightened detection resulting from utilisation of CT scans in the evaluation of children with complicated pneumonia. There was a consistent increase in CT scans performed at the present authors' institution starting in the early 1990s and preceding the detection of the first cases of NP. Since CT scans are the standard mode for diagnosis of NP, the increased use of CT would increase detection of NP cases. During the same time period, there was not a similar increase in the number of admissions for pneumonia to the institution. It lends itself to reason, therefore, that the observed increase of NP detection parallels the increases observed in complicated parapneumonic effusions in recent years $[7,14,15]$.

The increase in NP cases over time may also be due to a changing spectrum of causative organisms, as has been postulated as the underlying cause of the rise in complicated pneumonia in general. Pneumococcus spp. was the predominant organism detected in the present study, similar to most previous reports of NP [1-4]. Due to the retrospective nature of the present study, it was impossible to identify the specific Pneumococcal spp. serotypes in the patients. Thus, the present authors were unable to replicate the analysis performed by TAN et al. [14] and comment on secular changes of particular strains and their relationship to the introduction of the

\section{TABLE 3 Necrotising pneumonia outcomes and management during hospitalisation}

\section{Summary statistics}

Subjects

$\begin{array}{lcc}\text { Hospital management } & & \\ \text { Length of stay days } & 12(9-17) & 80 \\ \text { Length of fever days } & 6(3-9) & 71 \\ \text { Length of total antibiotic therapy days } & 28(20-40) & 80 \\ \text { Intensive care unit duration days } & 4(2-10) & 25 \\ \text { Length of pleural drainage days } & 6(4.5-9.5) & 63 \\ \text { Length of follow-up days } & 174(77-360) & 63 \\ \text { Procedures performed for patients with pleural effusion } & & 69 \\ \text { Thoracentesis } & 16(23) & 69 \\ \text { Radiologically guided pigtail catheter placement } & 41(59) & 69 \\ \text { Chest tube placed } & 32(46) & 69 \\ \text { Thrombolysis } & 17(25) & 69 \\ \text { Video assisted thoracoscopic surgery } & 12(17) & 69 \\ \text { Partial lung resection } & 1(1) & 69 \\ \text { Open thoracotomy / pleural decortication } & 3(4) & 69 \\ \text { Chest drainage only } & 47(68) & 69 \\ \text { Chest drainage and surgery } & 16(23) & 69 \\ \text { Thoracentesis only } & 6(9) & 80 \\ \text { Complications } & & 80 \\ \text { Intensive care unit required } & 25(31) & 80 \\ \text { Bronchopleural fistula } & 10(13) & 80 \\ \text { Re-admissions } & 8(10) & 80 \\ \text { Mortality } & 0(0) & 1(1) \\ \text { Extracorporeal membrane oxygenation } & & \end{array}$




\begin{tabular}{|c|c|c|c|c|}
\hline $\begin{array}{l}\text { Assoc } \\
\text { diseas }\end{array}$ & $\begin{array}{l}\text { tween type of inte } \\
\text { hospitalisation }\end{array}$ & for patients with $p$ & I effusions and outcomes & d to evolution of \\
\hline & Thoracentesis only & Chest drainage only & Chest drainage and surgery & No effusion present \\
\hline Subjects $\mathbf{n}$ & 6 & 47 & 16 & 11 \\
\hline Age yrs & $4.1(2.7-6.2)$ & $3.8(2.7-7.3)$ & $3.8(2.0-4.8)$ & $2.8(1.8-6.2)$ \\
\hline With drain & & $6(4-11)$ & $6(6-9)$ & \\
\hline Length of fever days & $4(3-7)$ & $6(3-10)$ & $5(3-10)$ & $4.5(2-7)$ \\
\hline
\end{tabular}

pneumococcal vaccine, as a possible contributor to NP. However, an increase in the variety of pathogens in these patients was observed. A number of cases were caused by other Streptococcal spp. and Staphylococcal spp., which have been previously reported in individual cases to cause NP [21, 22]. Several cases were also found to be caused by Fusobacterium spp. and $P$. aeruginosa, two bacterial species that have not been previously associated with childhood NP. Finally, three cases were caused by MRSA in 2003 and 2004. MRSA has been reported in one other case report of childhood NP [23] and as an emerging organism in childhood empyema [15]. The overall impact of the expanding range of causative organisms on both short- and long-term outcomes in NP warrants further study and 42 of the present cases had no identified microbiological cause. None of the patients with positive culture results had received $>3$ days of antibiotic pretreatment. A likely explanation is that the infection triggered a severe inflammation that resulted in the development of NP but the antibiotics given prior to the present intervention were sufficient to sterilise the pleural fluid. However, an important alternative possibility is that the culture negative cases were caused by organisms such as anaerobes, viruses, Mycoplasma pneumoniae or Chlamydia pneumoniae, which have been reported to cause NP in children, though were not part of the routine

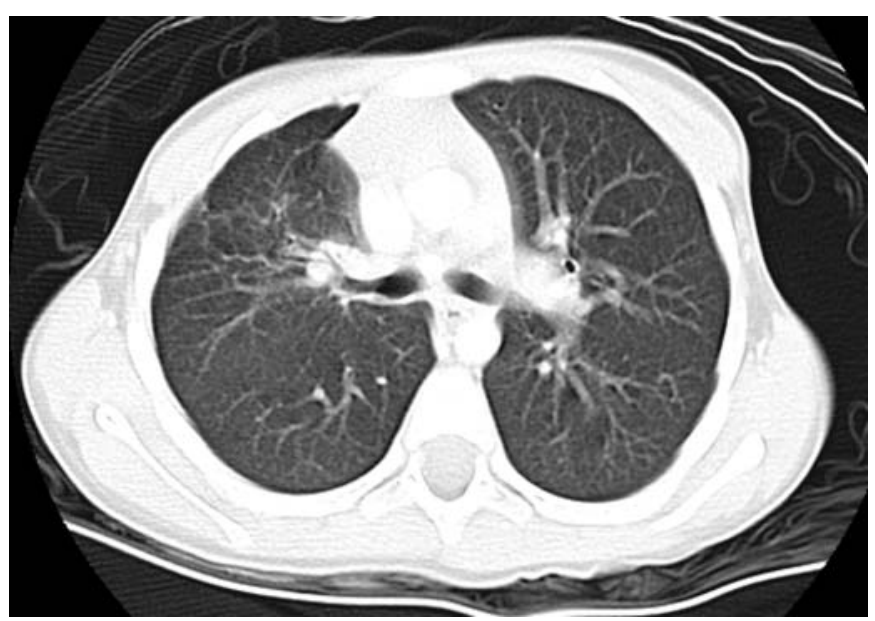

FIGURE 3. A repeat computed tomography scan on a 4-yr-old female obtained 9 months after initial presentation shows near-complete resolution of the parenchymal and pleural abnormalities with minimal parenchymal scarring 9 months after the hospitalisation. microbiological investigations in this series [12, 13, 24]. Such testing, using for instance PCR for virus or Mycoplasma spp. detection, could potentially increase the diagnostic yield in NP.

It is not possible to retrospectively perform rigorous evaluation of management strategies in NP, particularly because the frequent coexistence of empyema prevents uncoupling of the two conditions and separating the analyses of their respective contribution to overall morbidity. A difference in the length of pleural fluid drainage or length of fever was not observed, however, when comparing patients who had surgical intervention with those that were conservatively managed with nonsurgical chest drainage. The present authors propose that NP should be recognised as a distinct element of complicated pneumonia and, in particular, its presence should be considered as a separate complicating feature from the often accompanying pleural effusion. Diagnosis of NP requires CT imaging, and thus, such characterisation in patients with complicated pneumonia and continued symptoms despite appropriate medical therapy is recommended. The lack of recognition of NP can lead to erroneous diagnoses with serious errors in management. Based on the universally favourable outcomes with conservative management, the present authors do not agree that surgical resection for the treatment of NP is necessary, as has been implied in recent publications [25-27], and are concerned about the use of terminology, such as 'patients required' lung resection [14] and 'destroyed lung' [26]. The role of such interventions needs to be evaluated in prospective management studies of NP.

Children with NP have increased risk for developing a BPF. In a prior study of nine NP cases, five developed a BPF that was thought likely to be due to the friability of the inflamed pleura abutting the necrotised lung [28]. All patients who developed $\mathrm{BPF}$ in the present series had a chest drain in place for $>7$ days, raising the possibility that length of chest tube drainage is a risk factor for development of BPF. The present observations are, however, too few for a meaningful analysis.

The long-term outcomes for patients seen in follow-up have been good. All patients had resolution of clinical symptoms within 2 months of their hospitalisation. Although many children were too young for PFT, the children who had follow-up spirometry had essentially normal results. Performing PFT in these children could aid in further characterising any residual effects of NP over time. Follow-up chest radiographs and, in a few cases, CT scans have shown almost complete normalisation of pulmonary parenchyma within several months of hospitalisation (fig. 3). 
Overall, this pattern of improvement suggests that the lung damage caused by NP in children is transient. The radiographic resolution observed in the present cases confirms earlier similar reports in smaller cases series of NP [29]. Thus, when NP is diagnosed it should be recognised as a severe, yet, self-limiting and reversible disease.

In conclusion, necrotising pneumonia should be recognised as an increasingly detected complication of paediatric community-acquired pneumonia that is distinct from pleural effusion and empyema. Even though Pneumococcus spp. remains the most common causative bacterial pathogen, necrotising pneumonia in children can be caused by a variety of organisms including methicillin-resistant Staphylococcus aureus. Conservative management of necrotising pneumonia with antibiotics and chest drainage for pleural effusions results in good outcomes and there is no indication that surgical resection is necessary for the proper treatment of necrotising pneumonia. Despite the short-term severe morbidity of childhood necrotising pneumonia, long-term clinical outcomes are excellent with minimal resultant sequelae.

\section{ACKNOWLEDGEMENTS}

The authors would like to thank R. Malley and K. McIntosh (both Division of Infectious Diseases, Childrens Hospital Boston, Harvard Medical School, Boston, MA, USA) for critical review of the manuscript.

\section{REFERENCES}

1 Kerem E, Bar Ziv Y, Rudenski B, Katz S, Kleid D, Branski D. Bacteremic necrotizing pneumococcal pneumonia in children. Am J Respir Crit Care Med 1994; 149: 242-244.

2 Chen KC, Su YT, Lin WL, Chiu KC, Niu CK. Clinical analysis of necrotizing pneumonia in children: three-year experience in a single medical center. Acta Paediatr Taiwan 2003; 44: 343-348.

3 Hacimustafaoglu M, Celebi S, Sarimehmet H, Gurpinar A, Ercan I. Necrotizing pneumonia in children. Acta Paediatr 2004; 93: 1172-1177.

4 McCarthy VP, Patamasucon P, Gaines T, Lucas MA. Necrotizing pneumococcal pneumonia in childhood. Pediatr Pulmonol 1999; 28: 217-221.

5 Wong KS, Chiu CH, Yeow KM, Huang YC, Liu HP, Lin TY. Necrotising pneumonitis in children. Eur J Pediatr 2000; 159: 684-688.

6 Schadeck T, Beckers D, Eucher P, de Bilderling G, Tuerlinckx D, Bodart E. [Necrotizing pneumonia in children: apropos 4 cases.] Arch Pediatr 2006; 13: 1209-1214.

7 Ramphul N, Eastham KM, Freeman R, et al. Cavitatory lung disease complicating empyema in children. Pediatr Pulmonol 2006; 41: 750-753.

8 Hodina M, Hanquinet S, Cotting J, Schnyder P, Gudinchet F. Imaging of cavitary necrosis in complicated childhood pneumonia. Eur Radiol 2002; 12: 391-396.

9 Hsieh YC, Hsiao CH, Tsao PN, et al. Necrotizing pneumococcal pneumonia in children: the role of pulmonary gangrene. Pediatr Pulmonol 2006; 41: 623-629.

10 Curry CA, Fishman EK, Buckley JA. Pulmonary gangrene: radiologic and pathologic correlation. South Med J 1998; 91 : 957-960.
11 Hsieh YC, Hsueh PR, Lu CY, Lee PI, Lee CY, Huang LM. Clinical manifestations and molecular epidemiology of necrotizing pneumonia and empyema caused by Streptococcus pneumoniae in children in Taiwan. Clin Infect Dis 2004; 38: 830-835.

12 Oermann C, Sockrider MM, Langston C. Severe necrotizing pneumonitis in a child with Mycoplasma pneumoniae infection. Pediatr Pulmonol 1997; 24: 61-65.

13 Wang RS, Wang SY, Hsieh KS, et al. Necrotizing pneumonitis caused by Mycoplasma pneumoniae in paediatric patients: report of five cases and review of literature. Pediatr Infect Dis J 2004; 23: 564-567.

14 Tan TQ, Mason EO Jr, Wald ER, et al. Clinical characteristics of children with complicated pneumonia caused by Streptococcus pneumoniae. Pediatrics 2002; 110: 1-6.

15 Schultz KD, Fan LL, Pinsky J, et al. The changing face of pleural empyemas in children: epidemiology and management. Pediatrics 2004; 113: 1735-1740.

16 Wexler ID, Knoll S, Picard E, et al. Clinical characteristics and outcome of complicated pneumococcal pneumonia in a paediatric population. Pediatr Pulmonol 2006; 41: 726-734.

17 Klar A, Shoseyov D, Berkun Y, et al. Intestinal protein loss and hypoalbuminemia in children with pneumonia. J Pediatr Gastroenterol Nutr 2003; 37: 120-123.

18 Potts DE, Levin DC, Sahn SA. Pleural fluid $\mathrm{pH}$ in parapneumonic effusions. Chest 1976; 70: 328-331.

19 Heffner JE, Brown LK, Barbieri C, DeLeo JM. Pleural fluid chemical analysis in parapneumonic effusions. A metaanalysis. Am J Respir Crit Care Med 1995; 151: 1700-1708.

20 Mitri RK, Brown SD, Zurakowski D, et al. Outcomes of primary image-guided drainage of parapneumonic effusions in children. Pediatrics 2002; 110: e37.

21 Cengiz AB, Kanra G, Caĝlar M, Kara A, Güçer S, Ince T. Fatal necrotizing pneumonia caused by group A streptococcus. J Paediatr Child Health 2004; 40: 69-71.

22 Gillet Y, Issartel B, Vanhems P, et al. [Severe staphylococcal pneumonia in children.] Arch Pediatr 2001; 8: Suppl. 4, 742s-746s.

23 Al-Tawfiq JA, Aldaabil RA. Community-acquired MRSA bacteremic necrotizing pneumonia in a patient with scrotal ulceration. J Infect 2005; 51: e241-e243.

24 Tsai MH, Huang YC, Chen CJ, et al. Chlamydial pneumonia in children requiring hospitalization: effect of mixed infection on clinical outcome. J Microbiol Immunol Infect 2005; 38: 117-122.

25 Ayed AK, Al-Rowayeh A. Lung resection in children for infectious pulmonary diseases. Pediatr Surg Int 2005; 21 604-608.

26 Eren S, Eren MN, Balci AE. Pneumonectomy in children for destroyed lung and the long-term consequences. J Thorac Cardiovasc Surg 2003; 126: 574-581.

27 Kalfa N, Allal H, Lopez M, et al. An early thoracoscopic approach in necrotizing pneumonia in children: a report of three cases. J Laparoendosc Adv Surg Tech A 2005; 15: 18-22.

28 Hoffer FA, Bloom DA, Colin AA, Fishman SJ. Lung abscess versus necrotizing pneumonia: implications for interventional therapy. Pediatr Radiol 1999; 29: 87-91.

29 Donnelly LF, Klosterman LA. Cavitary necrosis complicating pneumonia in children: sequential findings on chest radiography. AJR Am J Roentgenol 1998; 171: 253-256. 